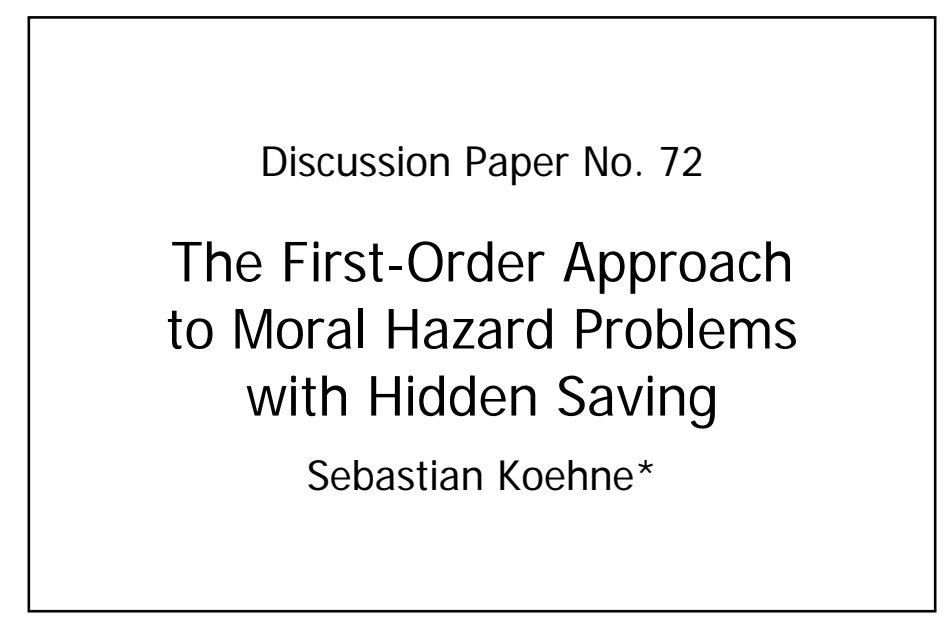

\begin{abstract}
August 2009
*Sebastian Koehne, Department of Economics, University of Mannheim, 68131 Mannheim, Germany, skoehne@mail.uni-mannheim.de
\end{abstract}

Financial support from the Deutsche Forschungsgemeinschaft through Graduiertenkolleg Risk and Liquidity in Financial, Commodity, and Factor Markets is gratefully acknowledged. 


\title{
The First-Order Approach to Moral Hazard Problems with Hidden Saving
}

\author{
Sebastian Koehne* \\ University of Mannheim
}

August 6, 2009

FIRST DRAFT

\begin{abstract}
This paper proposes a general method to validate the first-order approach for moral hazard problems with hidden saving. I show that strong convexity assumptions both on the agent's marginal utility of consumption and the distribution function of output arise naturally in this context. The first-order approach is valid given nonincreasing absolute risk aversion (NIARA) utility and log-convex distribution functions (LCDF) with monotone likelihood ratios (MLR). In a second step, I relax the LCDF condition by restricting the class of preferences and by imposing more structure on optimal wage schemes.
\end{abstract}

Keywords: principal-agent problems, moral hazard, hidden savings, first-order approach, log-convexity

JEL Classification: C61, D82, E21, H21

*Address: Department of Economics, University of Mannheim, 68131 Mannheim, Germany. Email: skoehne@mail.uni-mannheim.de. I would like to thank Ernst-Ludwig von Thadden, Nicola Pavoni, and especially Ian Jewitt for many helpful discussions. I received further helpful comments from Kaiji Chen, Marek Kapicka, Stefan Reichelstein, Richard Suen, Harald Uhlig, and conference participants at the Workshop on Public Economics 2009 Bonn. 


\section{Introduction}

The study of moral hazard problems is enormously simplified if one can use the first-order approach. By replacing the incentive constraint with the associated first-order condition, this approach allows the application of Lagrangian methods. The seminal works of Rogerson (1985) and Jewitt (1988) validate this procedure for the standard moral hazard problem. Very little is known, however, for more general moral hazard problems. In particular, the validity of the firstorder approach is not well understood for models in which the agent can secretly save (and borrow). This class of problems is rather important, since observability of the consumption-saving decision appears to be unrealistic for many interesting applications (employment relationships, insurance problems, taxation, etc).

As Kocherlakota (2004) points out, the validity of the first-order approach is significantly more complex in the presence of hidden saving 1 In addition to making sure that the agent's utility is at a global maximum with respect to the effort decision, one has to show the same for the saving decision, and most importantly for joint deviations to different effort and saving levels. Typically, the agent will combine a reduction of effort with an increased savings level to insure against the worsened output distribution. Therefore, ruling out joint deviations is the main difficulty in proving that first-order conditions are sufficient.

The present paper shows that conditions for the validity of the first-order approach can be derived quite easily nevertheless. The basic argument is simple. Suppose that output can take only two values. Then the agent's expected future consumption utility, depending on his choice of effort $e$ and saving $s$, takes the form

$$
p(e) u\left(c_{H}+s\right)+(1-p(e)) u\left(c_{L}+s\right),
$$

where $p(e)$ is the probability of the high output, and $c_{i}, i=H, L$, is the output-contingent transfer. To establish concavity of this expression, it is useful to rewrite expected utility as

$$
-(1-p(e))\left(u\left(c_{H}+s\right)-u\left(c_{L}+s\right)\right)+u\left(c_{H}+s\right) .
$$

\footnotetext{
${ }^{1}$ Kocherlakota (2004) provides an example in which the first-order approach to moral hazard with hidden saving fails even though the MLR and CDF conditions from Rogerson (1985) are satisfied. A similar argument shows that the conditions from Jewitt (1988) are also not sufficient for the problem with hidden saving.
} 
Only the first summand is a joint function of effort and saving, therefore this term is crucial. Because of the minus sign, we want to establish convexity of

$$
(1-p(e))\left(u\left(c_{H}+s\right)-u\left(c_{L}+s\right)\right) .
$$

The first factor, $(1-p(e))$, is the distribution function of output, evaluated at the low output level. The second factor, $\left(u\left(c_{H}+s\right)-u\left(c_{L}+s\right)\right)$, is closely related to the first derivative of the agent's utility function. Hence, to validate the first-order approach, we need suitable convexity properties of both the distribution function of output and the agent's marginal utility of consumption.

This reasoning extends to arbitrary output spaces and yields the following main conclusion: The first-order approach is valid if a) the agent has nonincreasing absolute risk aversion (NIARA) utility and b) the output technology has monotone likelihood ratios (MLR) and a log-convex distribution function (LCDF) 2 Note that LCDF requires more convexity than Rogerson's (1985) CDF condition and means that the (stochastic) returns to effort are strongly decreasing. In addition, NIARA makes sure that the agent's marginal utility of consumption is sufficiently convex.

In a second step, this paper explores how to relax the LCDF condition. One approach is to impose more convexity on the agent's marginal utility of consumption. A second approach is to impose more structure on the wage scheme, similar to the contribution by Jewitt (1988). As a key assumption, we need log-convexity of the primitive of the distribution function then. Even though this property is satisfied for some interesting examples in which LCDF fails, it is still relatively strong. For the standard moral hazard problem, by contrast, one only needs convexity (rather than log-convexity) of the primitive of the distribution function (Jewitt 1988, Condition 2.10a). That condition is more pleasing, since it is valid for all production functions with nonincreasing marginal returns to effort in each state of nature. On the other hand, of course, it is not surprising that the conditions derived in the present setup are more restrictive, given that hidden saving fundamentally challenges the first-order approach.

Previously, the first-order approach to moral hazard problems with hidden saving has only

\footnotetext{
${ }^{2}$ A function is called log-convex if the logarithm of that function is convex. Any log-convex functions is convex, but not vice versa.
} 
been examined under additional restrictions to the output technology or the agent's preferences. The pioneering work by Abraham and Pavoni (2009) imposes the spanning condition from Grossman and Hart (1983), whereas the paper by Koehne (2009) studies CARA utility. However, neither restriction is needed. In fact, neither restriction is particularly helpful, since the present findings contain the results by Abraham and Pavoni (2009) and Koehne (2009) as special cases. Moreover, the present findings point out what really drives the validity of the first-order approach: the combined degree of convexity of the distribution function and of the agent's marginal utility of consumption.

The first-order approach produces a very useful characterization of optimal contracts. Questions on the monotonicity of consumption or the value of information can be answered immediately, and one finds many analogies to the model without hidden saving. One also finds important differences between the two models as Abraham and Pavoni (2009) describe in detail. In particular, they show that hidden saving tends to make optimal contracts more convex. This implies that the associated tax-transfer scheme is typically more regressive than in the standard setup.

The first-order approach is also important because it gives the multi-period problem a tractable recursive structure, as discussed by Werning (2001, 2002), Kocherlakota (2004), and Abraham and Pavoni (2008), among others. Analytical results for the validity of the first-order approach provide a theoretical foundation for this procedure. The present paper is just a first step, however, because the generalization from two periods to the multi-period case is not trivial.

The paper proceeds as follows: Section[2 describes the setup of the model. Section 3 validates the first-order approach given NIARA, MLR and LCDF. Section 4 shows how to relax the latter assumption. Section 5 collects all proofs. Section 6 concludes.

\section{Setup}

I study a two-period principal-agent problem. In the first period, the agent makes a hidden saving decision. In the second period, the agent exerts a hidden work effort. Contracts are signed at the beginning of the first period and there is no renegotiation. To make the proofs a bit less cumbersome, I suppose that the distribution of output is absolutely continuous. All results go through for discrete output spaces as well. 


\subsection{Preferences}

The Principal (P) maximizes expected profits. For simplicity, P's discount factor equals 1 . The Agent (A) has von-Neumann-Morgenstern preferences and maximizes the expected value of

$$
u\left(c_{1}\right)+\beta\left(u\left(c_{2}\right)-v(e)\right),
$$

where $c_{t}$ denotes consumption and $e$ represents effort. Consumption utility $u$ is twice continuously differentiable and satisfies $u^{\prime}>0, u^{\prime \prime}<0$. Effort disutility $v$ is twice continuously differentiable and satisfies $v^{\prime}>0, v^{\prime \prime} \geq 0$.

\subsection{Technology}

In the first period, $\mathrm{A}$ is endowed with $w_{0}$ units of the consumption good and can save at zero interest. Negative saving, i.e., borrowing, is allowed. The set of feasible saving choices is the real interval $J$. The interval may be bounded or unbounded 3 A's saving decision is not observable.

In the second period, A exerts an unobservable work effort $e \in I$, where $I$ is a real interval. This generates a publicly observable stochastic output $x \in[\underline{x}, \bar{x}]$. The output is distributed according to the probability density $f(x, e)$, which is continuously differentiable and has full support for all $e \in I$.

\subsection{Contracts}

At the beginning of the first period, $\mathrm{P}$ proposes a contract $(w(\cdot), e, s)$ consisting of an outputcontingent wage scheme $w(\cdot)$ and recommended choices $(e, s)$. A's utility from rejecting the contract and saving optimally is $\underline{U}$. The contract is called optimal if it maximizes expected profits subject to the incentive compatibility constraint and the participation constraint, i.e., if

\footnotetext{
${ }^{3}$ The interval $J$ may be bounded below due to a borrowing constraint and bounded above due to a nonnegativity constraint.
} 
it solves the following problem:

$$
\begin{aligned}
& \max _{w(\cdot), e, s} \int_{\underline{x}}^{\bar{x}}(x-w(x)) f(x, e) d x \\
& \text { s.t. } \\
& (e, s) \in \underset{\left(e^{\prime}, s^{\prime}\right) \in I \times J}{\operatorname{argmax}} u\left(w_{0}-s^{\prime}\right)+\beta \int_{\underline{x}}^{\bar{x}} u\left(w(x)+s^{\prime}\right) f\left(x, e^{\prime}\right) d x-\beta v\left(e^{\prime}\right) \\
& u\left(w_{0}-s\right)+\beta \int_{\underline{x}}^{\bar{x}} u(w(x)+s) f(x, e) d x-\beta v(e) \geq \underline{U}
\end{aligned}
$$

\subsection{First-order approach}

Problem (IP1) is extremely intricate. The incentive constraint (IC) consists of a two-dimensional continuum of inequalities. For all $e^{\prime} \in I, s^{\prime} \in \mathbb{R}$, it requires

$$
\begin{gathered}
u\left(w_{0}-s\right)+\beta \int_{\underline{x}}^{\bar{x}} u(w(x)+s) f(x, e) d x-\beta v(e) \\
\geq u\left(w_{0}-s^{\prime}\right)+\beta \int_{\underline{x}}^{\bar{x}} u\left(w(x)+s^{\prime}\right) f\left(x, e^{\prime}\right) d x-\beta v\left(e^{\prime}\right) .
\end{gathered}
$$

To obtain a problem that can be solved by standard methods, one replaces the incentive constraint by the agent's first-order necessary conditions. This gives rise to the following problem:

$$
\begin{aligned}
& \max _{w(\cdot), e, s} \int_{\underline{x}}^{\bar{x}}(x-w(x)) f(x, e) d x \\
& \text { s.t. } \\
& \beta \int_{\underline{x}}^{\bar{x}} u(w(x)+s) f_{e}(x, e) d x-\beta v^{\prime}(e)=0 \\
& u^{\prime}\left(w_{0}-s\right)-\beta \int_{\underline{x}}^{\bar{x}} u^{\prime}(w(x)+s) f(x, e) d x=0 \\
& u\left(w_{0}-s\right)+\beta \int_{\underline{x}}^{\bar{x}} u(w(x)+s) f(x, e) d x-\beta v(e) \geq \underline{U}
\end{aligned}
$$

Solutions to ( $(\mathrm{P2})$ are denoted by $\left(w^{*}(\cdot), e^{*}, s^{*}\right)$. The associated consumption levels are denoted by $c_{0}^{*}=w_{0}^{*}-s^{*}$ and $c^{*}(x)=w^{*}(x)+s^{*}$.

Replacing the true problem ( $(\mathrm{P} 1)$ by the first-order problem $(\overline{\mathrm{P} 2})$ is a valid procedure only if their solutions coincide. Assuming that the solutions to (P1) are interior with respect to effort and saving, this will be the case if and only if the contracts solving ( $(\mathrm{P} 2)$ are incentive compatible. 
A sufficient condition for incentive compatibility is that the agent's decision problem is concave at these contracts. The remainder of this paper will identify conditions under which this is the case.

\section{A sufficient condition for concavity of the agent's problem}

In this section, I validate the first-order approach using nonincreasing absolute risk aversion, monotonicity of the wage scheme, and an assumption on the curvature of the output distribution function. This procedure strengthens the classic approach of Mirrlees (1979) and Rogerson (1985).

Using $\lambda, \mu$ and $\xi$ as the Lagrange multipliers associated with the constraints ( $(\overrightarrow{\mathrm{PC}})$, ( $\mathrm{FOCe})$, $(\overline{\mathrm{FOCS}})$, respectively, the first-order condition of the Lagrangian of problem ( $\mathrm{P} 2)$ with respect to wages is

$$
0=-f\left(x, e^{*}\right)+\mu \beta u^{\prime}\left(c^{*}(x)\right) f_{e}\left(x, e^{*}\right)-\xi \beta u^{\prime \prime}\left(c^{*}(x)\right) f\left(x, e^{*}\right)+\lambda \beta u^{\prime}\left(c^{*}(x)\right) f\left(x, e^{*}\right), \quad x \in[\underline{x}, \bar{x}] .
$$

Equivalently,

$$
\frac{1}{\beta u^{\prime}\left(c^{*}(x)\right)}=\lambda+\mu \frac{f_{e}\left(x, e^{*}\right)}{f\left(x, e^{*}\right)}+\xi \alpha\left(c^{*}(x)\right), \quad x \in[\underline{x}, \bar{x}]
$$

where $\alpha(c)=-u^{\prime \prime}(c) / u^{\prime}(c)$ is A's coefficient of absolute risk-aversion.

Expression (6) equates the principal's costs and benefits of marginally increasing the agent's utility at output $x$, normalized by the probability density (Abraham and Pavoni 2009). Compared to the standard moral hazard problem, there is now the additional term $\xi \alpha\left(c^{*}(x)\right)$, because an increase of $u\left(c^{*}(x)\right)$ relaxes the agent's Euler equation 4

I will often use the following two assumptions to give equation (6) more structure.

MLR. The likelihood ratio function, $f_{e}(x, e) / f(x, e)$, is continuously differentiable and nondecreasing in output $x$ for all effort levels $e$.

\footnotetext{
${ }^{4}$ Note that an increase of $\beta u\left(c^{*}(x)\right)$ by one marginal unit costs the principal $1 /\left(\beta u^{\prime}\left(c^{*}(x)\right)\right)$ units of consumption. On the other hand, it generates a benefit of $\lambda$ because the participation constraint is relaxed and a benefit (or cost) of $\mu f_{e} / f$ because the incentive constraint is relaxed (or tightened). In addition, there is a benefit of $\xi \alpha\left(c^{*}(x)\right)$ because an increase of $\beta u\left(c^{*}(x)\right)$ mitigates the agent's wish to save (Abraham and Pavoni 2009).
} 
NIARA. The agent's coefficient of absolute risk aversion, $\alpha(c)=-u^{\prime \prime}(c) / u^{\prime}(c)$, is continuously differentiable and nonincreasing in consumption $c$.

MLR is standard and simply means that more output is indicative of higher effort. NIARA implies that the multipliers $\lambda, \mu, \xi$ in the Kuhn-Tucker condition (6) are positive: $\lambda>0, \mu>0$, $\xi>0$ (Abraham and Pavoni 2009). Moreover, MLR plus NIARA is sufficient for A's consumption scheme $c^{*}(x)=w^{*}(x)+s^{*}$ to be continuously differentiable and nondecreasing in output $x$; see equation (6) 5

As noted before, the first-order approach is valid if A's objective function

$$
(e, s) \mapsto u\left(c_{0}^{*}-s\right)+\beta \int_{\underline{x}}^{\bar{x}} u\left(c^{*}(x)+s\right) f(x, e) d x-\beta v(e)
$$

is concave in $(e, s)$ at the contracts that solve ( $(\overline{\mathrm{P} 2})$. One can restrict attention to A's secondperiod consumption utility as the next result shows.

Lemma 1. A's decision problem is concave in $(e, s)$ if $A$ 's second-period consumption utility

$$
(e, s) \mapsto \int_{\underline{x}}^{\bar{x}} u\left(c^{*}(x)+s\right) f(x, e) d x
$$

is concave in $(e, s)$.

By focusing on A's second-period consumption utility, I ignore the curvature generated by the effort disutility function and by the effect of saving on first-period utility. In principle, one could obtain more general results by including these two effects. However, these terms substantially reduce the tractability of the problem. Besides, the role of the effort disutility function is limited anyway, since effort units can always be normalized such that this function is linear.

The following lemma identifies a sufficient condition for concavity of (8).

Lemma 2. Suppose $c^{*}(\cdot)$ is continuously differentiable and nondecreasing. Suppose the distribution function of output, $F(x, e)$, is convex in $e$ and for all $x \in[\underline{x}, \bar{x}], e \in I, s \in J$, we have

$$
\frac{F_{e e}(x, e) F(x, e)}{\left(F_{e}(x, e)\right)^{2}} \frac{u^{\prime \prime \prime}\left(c^{*}(x)+s\right) u^{\prime}\left(c^{*}(x)+s\right)}{\left(u^{\prime \prime}\left(c^{*}(x)+s\right)\right)^{2}} \geq 1 .
$$

${ }^{5}$ NIARA can be relaxed. Equation (6) implies that $c^{*}(\cdot)$ is nondecreasing under MLR if $-\left(u^{\prime \prime \prime} u^{\prime}-\left(u^{\prime \prime}\right)^{2}\right) \leq$ $-u^{\prime \prime}(\beta \xi)^{-1}$. This requires that the coefficient of absolute risk aversion does not increase too quickly. 
Then A's second-period consumption utility is concave in $(e, s)$.

To understand condition (9), note that $F_{e e} F /\left(F_{e}\right)^{2}$ is nonnegative if and only if $F$ is convex in $e$, and at least 1 if and only $F$ is log-convex in $e 6$ Hence, $F_{e e} F /\left(F_{e}\right)^{2}$ measures the convexity of the distribution function $F$ as a function effort. This motivates the following concept.

LCDF. The distribution function of output, $F(x, e)$, is log-convex in effort $e$ for all output levels $x$.

A necessary but not sufficient condition for LCDF is that the distribution function is convex in effort. Hence, LCDF tightens the CDF condition from Mirrlees (1979) and Rogerson (1985).

To interpret LCDF, note that $F\left(x^{\prime}, e\right)$ equals $1-P\left(x>x^{\prime} \mid e\right)$. Therefore, stating that $F\left(x^{\prime}, e\right)$ is log-convex in effort (or highly convex, in other words) implies that the probability $P\left(x>x^{\prime} \mid e\right)$ is highly concave in effort. For this reason, LCDF requires that the (stochastic) returns to effort are strongly decreasing: The probability $P\left(x>x^{\prime} \mid e\right)$ that output is larger than some level $x^{\prime}$ is highly concave in the agent's effort choice $e$ for all values of $x^{\prime}$.

Analogous to the interpretation of $F_{e e} F /\left(F_{e}\right)^{2}$, note that $u^{\prime \prime \prime} u^{\prime} /\left(u^{\prime \prime}\right)^{2}$ is a measure of convexity of A's marginal utility of consumption. This measure is nonnegative if and only if $u^{\prime}$ is convex, and at least 1 if and only if $u^{\prime}$ is log-convex. Convexity of $u^{\prime}$ is typically referred to as nonnegative prudence. Log-convexity of $u^{\prime}$ is equivalent to

$$
\frac{u^{\prime \prime \prime} u^{\prime}-\left(u^{\prime \prime}\right)^{2}}{\left(u^{\prime}\right)^{2}} \geq 0
$$

This is the case if and only if

$$
\frac{d}{d c}\left(-\frac{u^{\prime \prime}(c)}{u^{\prime}(c)}\right) \leq 0
$$

Hence, log-convexity of $u^{\prime}$ is equivalent to NIARA.

The main result is a now direct consequence of these observations: MLR, NIARA and LCDF validate the first-order approach.

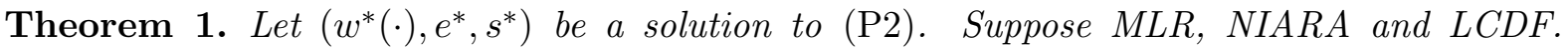
Then, given this contract, the agent's decision problem is concave in effort and saving. Hence, the contract is also a solution to (P1).

\footnotetext{
${ }^{6} \mathrm{~A}$ function is called log-convex if the logarithm of that function is convex. Any log-convex functions is convex, but not vice versa.
} 
While NIARA is not too problematic, LCDF is novel. Therefore some examples might be helpful.

Example 1 (Rogerson 1985). Rogerson's paper contains the following distribution function that is convex in effort and satisfies MLR:

$$
F(x, e)=\left(\frac{x}{\bar{x}}\right)^{e-\underline{e}}, \quad x \in[0, \bar{x}], e \in(\underline{e}, \infty)
$$

This distribution function is not only convex in $e$, but even satisfies LCDF. Note

$$
\log (F(x, e))=(e-\underline{e}) \log \left(\frac{x}{\bar{x}}\right)
$$

which shows that $F(x, e)$ is $\log$-linear in $e$ for all $i$.

Example 2 (Log-logistic distribution). Let $0<\beta \leq 1$. Consider the following distribution function:

$$
F(x, e)=\frac{1}{1+(e / x)^{\beta}}, \quad x \in[0, \infty), e \in(0, \infty) .
$$

It is not difficult to see that MLR is satisfied. Moreover, note

$$
\log (F(x, e))=-\log \left(1+(e / x)^{\beta}\right)
$$

Since $\beta \leq 1$, the expression $(e / x)^{\beta}$ is concave in $e$. Since the logarithm is increasing and concave, equation (15) shows that $\log (F(x, e))$ is convex in $e$. Thus, LCDF is satisfied.

The following examples apply to discrete output spaces $X=\left\{x_{1}, \ldots, x_{n}\right\}, x_{i}<x_{j}$ for $i<j$. In this setup, wages are vectors $\left(w_{1}, \ldots, w_{n}\right) \in \mathbb{R}^{n}$, and probability weights $\left(p_{1}(e), \ldots, p_{n}(e)\right)$ replace the density function $f(x, e)$. The previous results extend to the discrete setup without difficulty.

Example 3 (Two outputs). Consider the case with two possible outputs, $x_{L}<x_{H}$, and associated probabilities $p_{L}(e)=1-p(e), p_{H}(e)=p(e)$, for some increasing function $p$ with $0 \leq p(e) \leq 1$. Since $p$ is increasing, MLR is satisfied. LCDF is equivalent to the log-convexity of $1-p(e)$. One example that satisfies this condition is the function $p(e)=1-\exp (-f(e))$, where $f: I \rightarrow(0, \infty)$ is increasing and concave. 
Example 4 (Spanning condition). Let $\left(\pi_{1 h}, \ldots, \pi_{n h}\right),\left(\pi_{1 l}, \ldots, \pi_{n l}\right)$ be two probability distributions on $\left\{x_{1}, \ldots, x_{n}\right\}$ such that $\pi_{i h} / \pi_{i l}$ is nondecreasing in $i$. (This implies that $\pi_{h}$ first-order stochastically dominates $\pi_{l}$.) Let

$$
p_{i}(e)=\Gamma(e) \pi_{i h}+(1-\Gamma(e)) \pi_{i l}
$$

for some increasing function $\Gamma$, with $0 \leq \Gamma(e) \leq 1$. Monotonicity of $\Gamma$, combined with the fact that $\pi_{i h} / \pi_{i l}$ is nondecreasing, yields MLR. Note

$$
F_{i}(e)=F\left(x_{i}, e\right)=\sum_{j=1}^{i} p_{j}(e)=(1-\Gamma(e)) \sum_{j=1}^{i}\left(\pi_{i l}-\pi_{i h}\right)+\sum_{j=1}^{i} \pi_{i h} .
$$

First-order stochastic dominance implies $\sum_{j=1}^{i}\left(\pi_{i l}-\pi_{i h}\right) \geq 0$. Therefore, LCDF holds if $1-\Gamma(e)$ is log-convex. This requirement is equivalent to

$$
\frac{\left(\Gamma^{\prime}(e)\right)^{2}}{-\Gamma^{\prime \prime}(e)(1-\Gamma(e))} \leq 1
$$

which is exactly the condition under which Abraham and Pavoni (2009) validate the first-order approach for the spanning condition and NIARA utility. Their proof relies heavily on the spanning condition and there is no obvious way how it generalizes to the setting considered in this paper. Moreover, Abraham and Pavoni's reading of the property in (18) is that the Frisch elasticity of leisure must not be larger than one (Abraham and Pavoni 2009, p. 16). This does not capture the precise sense in which (18) tightens the CDF condition from Mirrlees (1979) and Rogerson (1985), in contrast to the interpretation offered here.

\section{How to relax LCDF}

The previous section has relied on a relatively strong assumption regarding the curvature of the output technology. I now explore three ways of relaxing that assumption. The first method restricts the class of preferences. Methods 2 and 3 use the shape of the wage scheme in more detail. Different from the first method, the obtained conditions are less easily translated into assumptions on exogenous variables. However, they are simple to check in practice. 


\subsection{DARA instead of NIARA}

Recall that LCDF is equivalent to $F_{e e} F /\left(F_{e}\right)^{2} \geq 1$, while NIARA is equivalent to $u^{\prime \prime \prime} u^{\prime} /\left(u^{\prime \prime}\right)^{2} \geq$ 1. If the latter expression is bounded away from 1 , then Lemma 2 can be used to relax LCDF. This yields the following result.

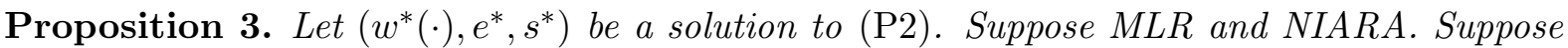
there exists a number $\eta>1$ such that for all $c$

$$
\frac{u^{\prime \prime \prime}(c) u^{\prime}(c)}{\left(u^{\prime \prime}(c)\right)^{2}} \geq \eta
$$

and for all $e \in I, x \in[\underline{x}, \bar{x}]$,

$$
\frac{F_{e e}(x, e) F(x, e)}{\left(F_{e}(x, e)\right)^{2}} \geq \frac{1}{\eta}
$$

Then, given this contract, the agent's decision problem is concave in effort and saving.

Note that (19) implies that the agent's preferences satisfy DARA: The coefficient of absolute risk aversion, $\alpha(c)=-u^{\prime \prime}(c) / u^{\prime}(c)$, is decreasing in consumption $c$. Moreover, note that (20) implies that $F_{e e}(x, e)$ is nonnegative. Hence, while (20) is weaker than LCDF, it still requires that the distribution function is convex in effort.

As an important example, consider CRRA utility: $u(c)=c^{1-\gamma} /(1-\gamma)$. Then we have

$$
\frac{u^{\prime \prime \prime}(c) u^{\prime}(c)}{\left(u^{\prime \prime}(c)\right)^{2}}=1+\frac{1}{\gamma}
$$

Hence, using Proposition 3, we conclude that the first-order approach is valid if for all $e \in I$, $x \in[\underline{x}, \bar{x}]$,

$$
\frac{F_{e e}(x, e) F(x, e)}{\left(F_{e}(x, e)\right)^{2}} \geq \frac{\gamma}{1+\gamma}
$$

Under the spanning condition from Example 4, for instance, this property is equivalent to

$$
\frac{\left(\Gamma^{\prime}(e)\right)^{2}}{-\Gamma^{\prime \prime}(e)(1-\Gamma(e))} \leq 1+\frac{1}{\gamma} \text { for all } e \in I
$$

This relaxes condition (18). 


\subsection{Concave wage schemes}

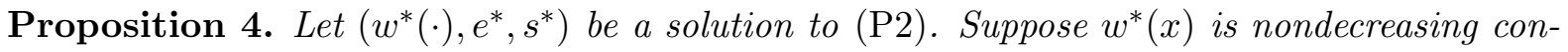
cave in output $x$. Suppose the distribution function of output, $F(x, e)$, is quasiconvex in $(x, e)$. Then, given this contract, the agent's decision problem is concave in effort and saving.

At first glance, Proposition 4 seems to suggest that the validity of the first-order approach is not affected by the introduction of hidden saving. Indeed, the result does not need any extra assumptions compared to the standard setup. However, there is a crucial difference between the two cases: The assumption of a concave wage scheme is relatively mild in the standard model, but much more restrictive in the present setting.

To see why, recall from the Kuhn-Tucker condition (6) that solutions to (르) are characterized by

$$
w^{*}(x)+s^{*}=g^{-1}\left(\lambda+\mu \frac{f_{e}\left(x, e^{*}\right)}{f\left(x, e^{*}\right)}\right)
$$

with $g(c)=1 /\left(\beta u^{\prime}(c)\right)-\xi \alpha(c)$. The following result argues that, given $\xi>0$, the function $g^{-1}$ is typically not concave. Thus, in the present setting, concave wage schemes are not guaranteed under the common assumption that the likelihood ratio function $f_{e}(x, e) / f(x, e)$ is concave in output $x$.

Consider utility functions of the HARA class,

$$
u(c)=\zeta\left(\eta+\frac{c}{\gamma}\right)^{1-\gamma}, \quad \eta+\frac{c}{\gamma}>0
$$

with $\zeta(1-\gamma) / \gamma>0$ and $\gamma>0.7$ Special members of this class are CRRA utility $(\eta=0)$ and CARA utility $(\gamma \rightarrow \infty)$.

Lemma 5. Suppose utility is of the class defined in (25). For $\xi=0$ (no hidden saving), the function $g^{-1}$ is concave if and only if $\gamma \geq 1$. For $\xi>0$ (hidden saving), the function $g^{-1}$ is concave if and only if utility is CARA.

\footnotetext{
${ }^{7}$ Condition $\zeta(1-\gamma) / \gamma>0$ ensures that $u$ is increasing and concave; condition $\gamma>0$ ensures NIARA.
} 


\subsection{Using the primitive of the distribution function}

The assumption that wages are concave in output is relatively strong as the previous subsection has shown. The present subsection relaxes LCDF under a condition that is somewhat weaker. To simplify the argument, I suppose that the wage scheme is twice continuously differentiable in output: 8

Given a mild assumption on the agent's preferences, concavity of the wage scheme is stronger than the following property.

Lemma 6. Suppose NIARA and suppose $-u^{\prime \prime \prime}(c) / u^{\prime \prime}(c)$ is nonincreasing in $c$. Then the condition

$$
-\frac{d^{2}\left(u\left(c^{*}(x)+s\right)\right)}{d x^{2}} \text { is log-convex in saving } s
$$

is necessary but not sufficient for $w^{*}(x)$ to be concave in output $x$.

The assumption that $-u^{\prime \prime \prime}(c) / u^{\prime \prime}(c)$ is nonincreasing in $c$ (nonincreasing absolute prudence) is innocuous. For instance, it is satisfied for all utility functions of the class defined in (25).

The property identified in Lemma 6 yields the following result.

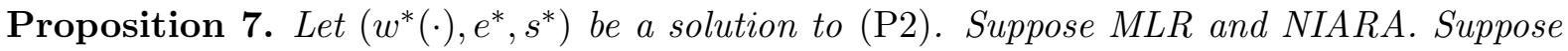
that for all output levels $x$

$$
\begin{aligned}
& -\frac{d^{2}\left(u\left(c^{*}(x)+s\right)\right)}{d x^{2}} \text { is log-convex in saving } s, \\
& \widetilde{F}(x, e)=\int_{\underline{x}}^{x} F(z, e) d z \quad \text { is log-convex in effort } e .
\end{aligned}
$$

Then, given this contract, the agent's decision problem is concave in effort and saving.

Note that log-convexity is preserved under integration (Boyd and Vandenberghe 2004, p. 106). Therefore, log-convexity of the primitive, LCP, is a weaker assumption than logconvexity of the distribution function, LCDF. Intuitively, the primitive $\widetilde{F}(x, e)$ will be logconvex in $e$ if the distribution function $F(x, e)$ is log-convex in $e$ for small values of $x$ and "not too misbehaved" for large values of $x$. In fact, $F(x, e)$ does not even have to be convex in $e$ as the following example shows.

\footnotetext{
${ }^{8}$ As the Kuhn-Tucker condition (6) shows, the wage scheme $w^{*}(x)=c^{*}(x)-s^{*}$ will be $C^{2}$ in $x$ if $f_{e}(x, e) / f(x, e)$ is $C^{2}$ in $x$ and $u^{\prime}(c), \alpha(c)$ are $C^{2}$ in $c$.
} 
Example 5 (Beta Prime distribution). Consider the Beta Prime distribution with parameter $b=2$ :

$$
f(x, e)=\frac{x^{e-1}(1+x)^{-e-2}}{B(e, 2)}, \quad x \in[0, \infty), e \in(0, \infty),
$$

where $B(e, b)$ represents the Beta function. The likelihood ratio function $f_{e}(x, e) / f(x, e)$ is nondecreasing concave in $x$, hence the class of preferences satisfying LCS is nonempty. The distribution function is

$$
F(x, e)=(1+e+x) x^{e}(1+x)^{-e-1} .
$$

It is easy to see that $F(x, e)$ is not convex in $e$ for all $x$. However, the primitive of the distribution function,

$$
\widetilde{F}(x, e)=x\left(\frac{x}{1+x}\right)^{e}
$$

is log-linear in $e$. Therefore, LCP is satisfied.

\section{Proofs}

Proof of Lemma 1. A's objective function is

$$
(e, s) \mapsto u\left(c_{0}^{*}-s\right)+\beta \int_{\underline{x}}^{\bar{x}} u\left(c^{*}(x)+s\right) f(x, e) d x-\beta v(e) .
$$

Since $u$ is concave, the first summand is concave in $(e, s)$. Since $v$ is convex, the third summand is concave in $(e, s)$.

Proof of Lemma Q Using partial integration, A's second-period consumption utility can be rewritten as

$$
\int_{\underline{x}}^{\bar{x}} u\left(c^{*}(x)+s\right) f(x, e) d x=u\left(c^{*}(\bar{x})+s\right)-\int_{\underline{x}}^{\bar{x}}\left(c^{*}\right)^{\prime}(x) u^{\prime}\left(c^{*}(x)+s\right) F(x, e) d x .
$$

Hence, A's second-period consumption utility is concave in $(e, s)$ if the function

$$
(e, s) \mapsto-\int_{\underline{x}}^{\bar{x}}\left(c^{*}\right)^{\prime}(x) u^{\prime}\left(c^{*}(x)+s\right) F(x, e) d x
$$


is concave, or equivalently if the function

$$
(e, s) \mapsto \int_{\underline{x}}^{\bar{x}}\left(c^{*}\right)^{\prime}(x) u^{\prime}\left(c^{*}(x)+s\right) F(x, e) d x
$$

is convex. We want to show that

$$
g(e, s ; x)=u^{\prime}\left(c^{*}(x)+s\right) F(x, e)
$$

is convex in $(e, s)$ for all $x$. Since $\left(c^{*}\right)^{\prime}(x) \geq 0$ by assumption, and since convexity is preserved under integration, this will imply convexity of (32).

The function $g(e, s ; x)$ is convex in $(e, s)$ if and only if its Hessian has a nonnegative diagonal and a nonnegative determinant. Omitting all arguments, the Hessian equals

$$
H=\left(\begin{array}{cc}
F_{e e} u^{\prime} & F_{e} u^{\prime \prime} \\
F_{e} u^{\prime \prime} & F u^{\prime \prime \prime}
\end{array}\right) .
$$

The first diagonal entry is nonnegative by assumption. Condition (9) is equivalent to the statement that the determinant of $H$ is nonnegative. In that case, the second diagonal entry of $H$ must also be nonnegative.

Proof of Theorem 1. By Lemma 1, it is sufficient to establish concavity of A's second-period consumption utility. Due to MLR and NIARA, the Kuhn-Tucker condition (6) implies that consumption $c^{*}(x)$ is continuously differentiable and nondecreasing in output $x$. Moreover, LCDF and NIARA imply that condition (9) from Lemma 2 is satisfied. Hence, A's secondperiod consumption utility is concave.

Proof of Proposition [3. Direct consequence of Lemma 1 and Lemma 2

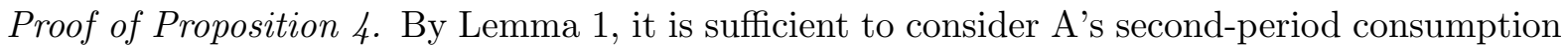
utility. Moreover, due to quasiconvexity of the distribution function, the output technology can be represented by a production function $x=\varphi(e, \epsilon)$, with $\varphi(e, \epsilon)$ nondecreasing concave in effort $e$ and nondecreasing in the stochastic state of nature $\epsilon$ (Jewitt 1988, Lemma 2). 
Using this representation, we can write A's second-period consumption utility as

$$
\int_{\underline{x}}^{\bar{x}} u\left(c^{*}(x)+s\right) f(x, e) d x=\mathbb{E}\left[u\left(c^{*}(\varphi(e, \epsilon))+s\right)\right],
$$

where $\mathbb{E}[\cdot]$ denotes expectations with respect to the state of nature $\epsilon$. Since $\varphi(e, \epsilon)$ is concave in $e$ and $c^{*}(x)=w^{*}(x)+s^{*}$ is nondecreasing concave in $x$, the composition $c^{*}(\varphi(e, \epsilon))$ is concave in $e$. Hence, the function $c^{*}(\varphi(e, \epsilon))+s$ is concave in $(e, s)$. Since $u$ is nondecreasing concave, and since concavity is preserved under integration, this completes the proof.

Proof of Lemma 5. First of all, since $g$ is nondecreasing, note that $g^{-1}$ is concave if and only if $g$ is convex. If $\xi=0$, then $g$ is convex if and only if $1 / u^{\prime}$ is convex. For HARA utility, this is equivalent to the condition $\gamma \geq 1$.

Now consider the case $\xi>0$. Verifying concavity of $g^{-1}$ for CARA is straighforward. Suppose that utility is not CARA, i.e., suppose $\gamma<\infty$. We have

$$
g(c)=\frac{1}{\beta u^{\prime}(c)}-\xi \alpha(c)=\frac{\gamma}{\beta \zeta(1-\gamma)}\left(\eta+\frac{c}{\gamma}\right)^{\gamma}-\xi\left(\eta+\frac{c}{\gamma}\right)^{-1}
$$

Hence, the second derivative of $g$ equals

$$
g^{\prime \prime}(c)=-\frac{1}{\beta \zeta}\left(\eta+\frac{c}{\gamma}\right)^{\gamma-2}-\frac{2 \xi}{\gamma^{2}}\left(\eta+\frac{c}{\gamma}\right)^{-3} .
$$

This is positive if and only if

$$
\frac{\gamma^{2}}{-\zeta \beta}\left(\eta+\frac{c}{\gamma}\right)^{\gamma+1} \geq 2 \xi
$$

The left-hand side in (38) goes to zero if $\eta+c / \gamma$ is small. Hence, (38) does not hold for all feasible consumption levels. In other words, $g$ is not convex. This implies that $g^{-1}$ is not concave.

Proof of Lemma 6. Suppose $w^{*}(x)$ is concave in $x$. Equivalently, $c^{*}(x)=w^{*}(x)+s^{*}$ is concave in $x$. The function in ( $(\underline{\mathrm{LCS}})$ can be represented as

$$
-\frac{d^{2}\left(u\left(c^{*}(x)+s\right)\right)}{d x^{2}}=\left(-\left(c^{*}\right)^{\prime \prime}(x)\right) u^{\prime}\left(c^{*}(x)+s\right)+\left(\left(c^{*}\right)^{\prime}(x)\right)^{2}\left(-u^{\prime \prime}\left(c^{*}(x)+s\right)\right) .
$$


The first summand in (39) is log-convex in $s$, since $-\left(c^{*}\right)^{\prime \prime}(x) \geq 0$ and since $u^{\prime}$ is log-convex due to NIARA. The second summand is log-convex in $s$, since $\left(\left(c^{*}\right)^{\prime}(x)\right)^{2} \geq 0$ and since $-u^{\prime \prime}$ is log-convex when $-u^{\prime \prime \prime} / u^{\prime \prime}$ is nonincreasing. Since log-convexity is preserved under summation (Boyd and Vandenberghe 2004, p. 105), the function in (LCS) is therefore log-convex in $s$.

On the other hand, suppose that the function in ([LCS) is log-convex in $s$. As (39) shows, this does not imply that $c^{*}(x)$ or $w^{*}(x)=c^{*}(x)-s^{*}$ is concave in $x$ in general.

Proof of Proposition 7 . As Lemma 1 shows, it is sufficient to establish concavity of

$$
(e, s) \mapsto \int_{\underline{x}}^{\bar{x}} u\left(c^{*}(x)+s\right) f(x, e) d x .
$$

This is equivalent to establishing convexity of

$$
(e, s) \mapsto-\int_{\underline{x}}^{\bar{x}} u\left(c^{*}(x)+s\right) f(x, e) d x
$$

Using two steps of partial integration, the latter function can be rewritten as

$$
\left.-u\left(c^{*}(\bar{x})+s\right)+\left(c^{*}\right)^{\prime}(\bar{x}) u^{\prime}\left(c^{*}(\bar{x})+s\right)\right) \widetilde{F}(\bar{x}, e)+\int_{\underline{x}}^{\bar{x}}\left(-\frac{d^{2}\left(u\left(c^{*}(x)+s\right)\right)}{d x^{2}}\right) \widetilde{F}(x, e) d x .
$$

First, note that the expression $-u\left(c^{*}(\bar{x})+s\right)$ is convex in $(e, s)$ due to the concavity of $u$. Moreover, the expression

$$
\left.\left(c^{*}\right)^{\prime}(\bar{x}) u^{\prime}\left(c^{*}(\bar{x})+s\right)\right) \widetilde{F}(\bar{x}, e)
$$

is convex in $(e, s)$ by an argument similar to Lemma 2. For the third term in (42), note that

$$
-\frac{d^{2}\left(u\left(c^{*}(x)+s\right)\right)}{d x^{2}} \widetilde{F}(x, e)
$$

is the product of a function that is $\log$-convex in $s$ and a function that is $\log$-convex in $e$. Such products are convex in $(e, s)$ as one easily verifies. Since convexity is preserved under integration, the third term in (42) is thus convex as well. This completes the proof. 


\section{Concluding remarks}

This paper proposes a general method to validate the first-order approach for moral hazard problems with hidden saving. For this question, the key challenge is to rule out joint deviations to lower effort and higher saving levels. I use convexity assumptions on the agent's marginal utility of consumption and the distribution function of output to limit the gains of such deviations. An important open question is how these results extend to the multi-period case. This difficult task is left for future research.

\section{References}

Abraham, A., And N. PAvoni (2008): "Efficient Allocations with Moral Hazard and Hidden Borrowing and Lending: A Recursive Formulation," Review of Economic Dynamics, 11(4), 781-803.

(2009): "Optimal Income Taxation and Hidden Borrowing and Lending: The First-Order Approach in Two Periods," University College London, January 2009. Mimeo. http://www.ucl.ac.uk/ uctpnpa/FOC.pdf.

Boyd, S., And L. Vandenberghe (2004): Convex optimization. Cambridge University Press.

Grossman, S. J., and O. D. Hart (1983): "An Analysis of the Principal-Agent Problem," Econometrica, 51(1), 7-45.

Jewitt, I. (1988): "Justifying the First-Order Approach to Principal-Agent Problems," Econometrica, 56(5), 1177-1190.

Kocherlakota, N. R. (2004): "Figuring out the impact of hidden savings on optimal unemployment insurance," Review of Economic Dynamics, 7(3), 541-554.

Koenne, S. (2009): "The First-Order Approach to Moral Hazard Problems with Hidden Saving: The Case of CARA Utility," University of Mannheim, March 2009. Mimeo.

MirrleEs, J. (1979): "The Implications of Moral Hazard for Optimal Insurance," Seminar given at Conference held in honor of Karl Borch, Bergen, Norway. Mimeo. 
Rogerson, W. P. (1985): "The First-Order Approach to Principal-Agent Problems," Econometrica, 53(6), 1357-1367.

Werning, I. (2001): "Repeated Moral-Hazard with Unmonitored Wealth: A Recursive FirstOrder Approach," MIT. Mimeo. http://econ-www.mit.edu/files/1264.

_ (2002): "Optimal Unemployment Insurance with Unobservable Savings," MIT. Mimeo. http://econ-www.mit.edu/files/1267. 